ORIGINAL ARTICLE

\title{
Factors contributing to uncertainty in decision making and strategies to reduce this in undergraduate medical students.
}

\author{
Shahmain Shahzad ${ }^{1}$, Fahad Ismail ${ }^{2}$
}

Article Citation: Shahzad S, Ismail F. Factors contributing to uncertainty in decision making and strategies to reduce this in undergraduate medical students. Professional Med J 2022; 29(2):240-250. https://doi.org/10.29309/TPMJ/2022.29.02.6334

\begin{abstract}
Objectives: To identify the common factors that contribute towards uncertainty in decision making in undergraduate medical students and highlights the strategies that can be used to reduce this uncertainty. Study Design: Qualitative Study. Setting: Riphah International University, Avicenna Medical and Dental College, Lahore. Period: July 2020 to September 2020. Material \& Methods: Four focus groups were conducted with a total of 26 participants (2 with faculty members, 2 with final year students). Data was transcribed and analyzed using ATLAS. ti software. Thematic analysis was done. Results: Two main themes were identified: faculty related and student related reasons and strategies. The most common reason for uncertainty in decision making was a lack of knowledge while the most common strategy to reduce this uncertainty was increased patient exposure and enhancing medical knowledge. Conclusion: Uncertainty is ingrained in medical education and is commonly experienced by medical students during their interaction with patients. It is essential to identify the reasons that contribute to this uncertainty in decision making and highlight strategies that reduce it in order to foster competent and confident physicians.
\end{abstract}

Key words: $\quad$ Clinical Decision Making, Medical Students, Oral Case Presentations, Uncertainty.

\section{INTRODUCTION}

Uncertainty is a ubiquitous problem that has attracted growing attention in healthcare. ${ }^{1}$ It refers to a state of limited knowledge or a condition that lacks firm predictability; it is a metacognition, characterized by self-awareness of inadequate knowledge. As an intrinsic aspect of contemporary medicine, uncertainty can be encountered at any stage of medical consultation and undergraduate medical students often face uncertainty in decision making during their oral case presentations. In the medical field, uncertainty can be categorized into different dimensions, such as, case complexity, insufficient patient information and unpredictability regarding the course of illness and its outcomes. ${ }^{2}$

In healthcare, uncertainty commonly emanates from inadequate medical knowledge, limitations in scientific evidence, inaccurate test interpretation, lack of rapport development with the patient and biological variability. ${ }^{3}$ Other factors include: outdated medical information; patient data that is contributory but not discriminating enough; contradictory information; when key features do not match the diagnosis and when there is a lack of evidence for a course of action. ${ }^{4}$ While work has been done with regard to physicians, there is scarce data about reasons leading to uncertainty in undergraduate medical students.

There are many practices in medical education that facilitate the transformation of students into physicians, problem oriented patient presentation being the most essential as it allows interaction between the patient, medical team and medical students. Students engage in the system of cognitive apprenticeship where they learn how to present patients coherently with hands-on clinical training in their last two clinical years. ${ }^{5}$
1. BDS, MHPE, Demonstrator Medical Education, Avicenna Medical and Dental College, Lahore. 2. BDS, Demonstrator Prosthodontics, Avicenna Medical and Dental College, Lahore.

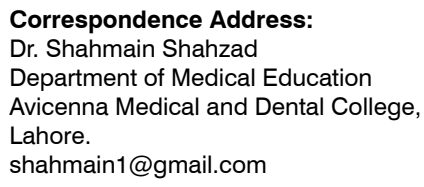


Medical students often face uncertainty during their clerkship due to their limited knowledge which can be a cause of severe stress. ${ }^{6,7}$ These students are seen to exhibit negative attitudes and are perceived as less confident, less competent and less engaged during clinical reasoning and decision making. ${ }^{8}$

There is a need to re-examine the concept of uncertainty in medical students due to the detrimental reactions it produces; it can cause significant anxiety, feelings of inadequacy, disillusionment, insecurity regarding professional skills, self-doubt and frustration. ${ }^{9}$ When faced with self-doubt, students feel unsure about their abilities and skills which are required to achieve something; it is directly associated with selfefficacy and self-esteem. Self-doubt increases academic procrastination which in turn decreases students' self-esteem. ${ }^{10}$ In order to avoid this negative impact on students and patients, it is essential to identify the common factors that contribute towards uncertainty and the strategies that can be incorporated to reduce uncertainty in decision making.

Uncertainty in medical practice is not only viewed as a problematic and challenging, but also as a serious concern because of the negative ways it affects the responsibilities and work of clinicians as well the fate of the patients. ${ }^{11}$ Physicians are often reluctant to express uncertainty to patients as they believe that it can overwhelm and confuse patients; they also have a fear of being sued by patients and losing their confidence. ${ }^{12}$ This reluctance leads to a variability in physician practice patterns, with an increase in hospital admissions, excessive ordering of diagnostic tests and empiric treatment regiments, increased referrals and an excessive use of other resources. Consequently, this puts patients at risk for undergoing adverse effects from unnecessary diagnostic tests and treatments, further increasing health care costs. ${ }^{9}$ It also increases unnecessary distress and dissatisfaction in patients, complicating quality of care standards, informed consent and malpractice judgements.

\section{MATERIAL \& METHODS}

This study was qualitative in nature. Qualitative study design helps the researcher to explore and understand the experiences of participants by delving into their perceptions. ${ }^{13}$ Data was collected through focus group discussions; four focus groups were conducted, out of which two were with final year medical students and two with faculty members teaching final year students. The first FGD was done at Avicenna Medical and Dental College, while the remaining three were done on Zoom software. This study was done over a period of two months after the ethical approval was given (Riphah/IRC/20/127).

Purposive sampling technique was employed. It is a non-random technique where a deliberate choice is made for selection of participants based on the qualities they possess and their willingness to provide information. Since the objective of this study was to explore factors associated with uncertainty in decision making and strategies to reduce this uncertainty, the focus was on both faculty members and students.

The sample size consisted of 26 participants, selected after applying the inclusion criteria, out of which 13 ( 9 males, 4 females) were final year MBBS students and 13 (6 males, 7 females) were faculty members. These faculty members were involved in teaching (lectures, tutorials, small group discussions) undergraduate medical students and all were actively involved in conducting long case presentations in clinical wards, having at least 5 years of relevant experience. They belonged to different institutions in Lahore and Islamabad/Rawalpindi which brought about a variety of experiences altogether.

The open-ended questions were formulated after an extensive literature search and latervalidated by 5 experts. These questions facilitated interaction where all participants shared their observations and personal experiences. Confidentiality and anonymity were ensured and informed consent was sought. The discussion was recorded and each discussion lasted from 60-90 minutes each. The data was carefully transcribed so as to prevent distortion of the meaning of data. 
The transcripts were then analyzed, followed by coding, using the software ATLAS.ti. A comparison of codes was made and redundant codes were removed. These were then merged into major themes. Thematic analysis refers to a data analysis method whereby data is analyzed and repeated patterns are noted; it is considered as a compelling analytical method for qualitative studies. ${ }^{14}$

\section{RESULTS}

Participants were first asked about the factors that influence uncertainty in decision making, followed by strategies that could help reduce uncertainty in clinical decision making in medical students. Two major themes emerged from the data for both research questions: student related factors and faculty related factors.

\section{Student Related Factors}

In student related factors, lack of knowledge seemed to be the most common reason, endorsed by majority of the respondents in all four focus groups.

Respondent T (faculty): 'I think the main factor is lack of knowledge; by this I don't mean students don't study, some students are very studious but are uncertain because their background system is not that developed for them to identify it and be certain immediately.'

Respondent UK (faculty): we've observed it can be due to the lack of knowledge or preparation for the exam or lack of exposure in the learning environment.'

Respondent M (student): 'So, one doesn't have enough knowledge of theory which causes uncertainty and inability to answer properly.'

This was followed by a lack of adequate preparation, student effort and lack of interest which led to inadequate clinical practice, eventually resulting in uncertainty in decision making. Faculty members also believed students did not take their ward rotations as seriously as they should have.
Respondent M (student): 'Another reason can be if I haven't reviewed the case properly, for example when I take the history and then immediately go to present; in those situations, I am uncertain as I have not prepared and cannot give an answer.'

Respondent S (faculty): 'Inadequate clinical practice and not taking the wards seriously. Wards start in third year but students usually do not go to the wards seriously until final year.'

Respondent AA (faculty): 'They aren't interested in doing anything themselves. You have to force them to take histories and make them stand in front of patients, they just don't want to do it and try to get away with it.

Faculty members were of the view that personality of students had a strong influence on uncertainty in their decision-making skills. Some students were confident enough and could exhibit certain behaviors that got them away in most situations. However, there were also students who despite studying and making an effort were unable to articulate themselves and showed hesitance in initiating conversation, posing them as uncertain in decision making.

Respondent FM (faculty): 'Personality matters a lot; there are some people who have studied a lot and then even know the correct answers but they are still not certain. They hesitate to commit anything; they are always uncertain. This goes from student life up to professional life.'

Respondent F (faculty): 'Some people have very anxious personalities who are panicky and they exhibit anxious expressions while some are confident with no such issues.'

Other reasons related to students were lack of attention in clinical wards, lack of presentation skills, inability to apply the knowledge learnt in lectures, high stake examinations, fear of examiner and fear of being wrong, stage fright, lack of training, practice of studying just a few weeks before the final exam, fear of embarrassment in front of colleagues, noisy environment, lack of patient cooperation and previous exposure 
to the same illness/examiner. Language barrier also contributed to uncertainty as some students from government schools were unable to grasp important concepts but understood well when explained in the Urdu language.

Respondent FM (faculty): 'When stakes are high, you are afraid to make mistakes which gives way to uncertainty.'

Respondent T (faculty): the second reason I think is the fear of being wrong, that is, embarrassment of giving the incorrect answer in front of the teacher and third is embarrassment in front of colleagues.'

Respondent UU (faculty): some people have fear of examiner, some examiners have notorious reputation so students are confused and uncertain in front of them...'
Respondent F (faculty): 'I have observed students especially from government colleges have a problem understanding English. If we explain the same topic in Urdu, they understand very well, but since our lectures and bedside long cases are presented in English, students are unable to grasp the concept.'

Respondent T (student): In case the patient is not cooperating or is not answering the question or is fidgety or tired so that obviously increases the uncertainty.'

Respondent WR (faculty): in our system there is a trend of studying at the end where they try to cover up for everything; they don't study the entire year but study in the last month or two. In this situation, students might gain some knowledge by cramming but they don't develop that grip.

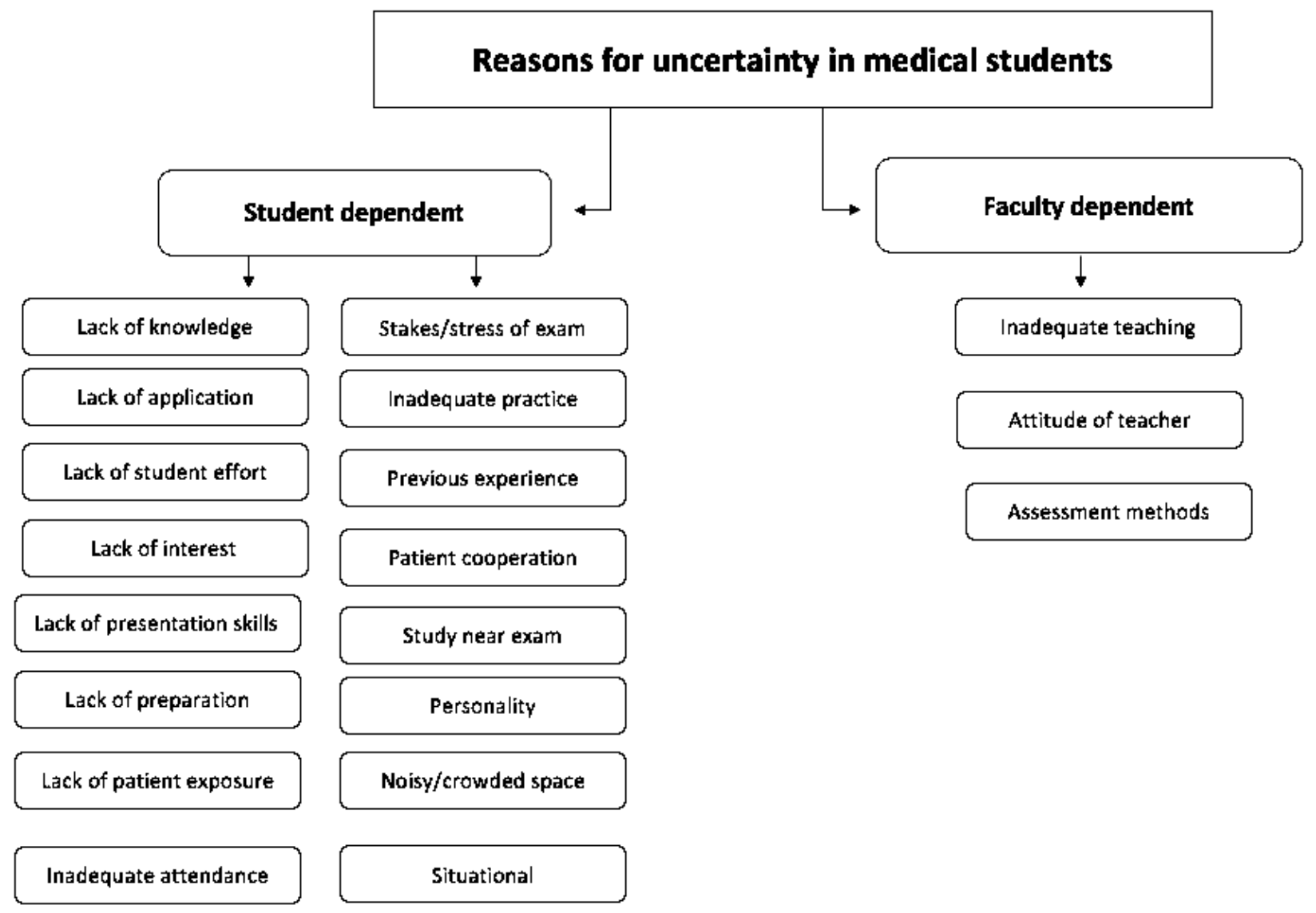

Figure-1. Reasons for uncertainty summarized. 


\section{Faculty Related Factors}

Inadequate teaching on part of faculty members, attitude of faculty/examiner and quality of teaching were associated with uncertainty in medical students. Instead of increasing exposure to patients, faculty members admitted they encouraged memorization as this is what the students were assessed on, essentially.

Respondent F (student): 'If we see the consultant or examiner giving their own implicit reactions such as pursing their lips or shaking their head or smirking that could make us feel as students that we might not be going in the correct way or we might not be saying the right things; that would shatter our confidence a little bit.'

Respondent K (student): 'Sometimes it's because of the consultant's attitude and behavior; some of them are really harsh and you can be very scared about what their reaction might be in case you give the wrong answer.'

Respondent AA (faculty): 'We make them sit in the tutorial room teaching them and hammering them all the time, which is very different than the clinical experience the lack is basically from our side as well, so it starts from that point that they haven't been trained to go to the patient in the ward and to talk to them, to interact with them, to take their history so they can develop their confidence and develop rapport with the patient.'

Respondent KMN (faculty): 'We are not examining the student; we're examining the books. That's the reason, that's what causes the uncertainty when they're taught one thing and asked something else.

\section{Strategies to reduce uncertainty in decision making.}

This answered the second sub research question that included all the strategies that can reduce uncertainty in decision making in undergraduate medical students. A total of 30 codes were identified, out which 10 were student related and 20 were faculty related as seen in (Table-I).
Student Dependent

Increase knowledge.

Enhance

communication skills.

Increased practice.

Learn from different

resources.

Learn with friends.

Initiate effort.

Peer assisted learning.

Increase exposure.

Improve personality.
Be regular in wards.

Faculty Dependent

Give hints/cues/guidance.

Be lenient.

Situational leadership.

Encourage reflection.

Counselling.

Mentorship.

Create positive learning

environment.

Avoid gestures to increase

uncertainty.

Identify and focus on weak

areas.

Have corrective attitude.

Interactive teaching.

Be more receptive.

Be polite/calm/patient.

Positive reinforcement.

Provide extra help.

Give constructive feedback.

Encourage students/

welcoming body language.

Avoid negative comments/

humiliation.

Improve training.

Prepare students from exam

point of view.

Table-I. Strategies to reduce uncertainty in decision making.

\section{Student Dependent Strategies}

The most common strategy to reduce uncertainty was increasing knowledge of students. This was given by both faculty and students as majority felt students would be more certain with acquisition of knowledge, which could only be done by students committing to take the initiative and put in the effort. Next to this, was a need to increase exposure to patients which would result in increased practice and rehearsal. This would help students to be more certain and confident about their decision making.

Respondent $A Q$ (student): by increasing our knowledge about the disease and clinical practice of long cases, increase the number of patients you see, improve history taking skills and improve your public speaking skills; this also counts as communication is improved.

Respondent $F$ (student): we need to brush up on our knowledge and as they say practice makes perfect, so we do have to go and practice 
repeatedly on patients and maybe take a friend along.

Respondent AM (student): 'Practice and exposure. For professional competence, practice is necessary; more time in the wards.

Two students also mentioned how simulation activities with their fellow peers helped them learn better and enhance their communication skills. Another suggestion came from a faculty member of adopting the concept of peer assisted learning as some students don't feel comfortable asking teachers questions.

Respondent TZ (student): what has helped me a lot is asking my friends to do a sort of a simulation with me, where sometimes I would become the medical student and l'd ask my friend 'you pretend to be the patient, l'll be the student, lets interact and imitate a medical case and discuss it with each other so that this would obviously be better for our communication skills.

Respondent UU (faculty): 'I also recommend peer assisted learning because some students are not comfortable with their seniors or teachers so they can learn from their peers.'

\section{Faculty Dependent Factors}

The most commonly mentioned answer was hints, cues or guidance from faculty members in case the students had difficulty presenting the case. Most students agreed with this and were of the view that often hints from faculty members and their receptive attitude steered them in the right direction.

Respondent AA (faculty): we even give them hints so they know what the topic is so they can discuss it or think about it. And if that doesn't work, we give them another hint, so basically trying to put them on the track that you should start from here.

Respondent S (student): faculty should definitely help us, they should guide us about how we should go about it, if they give us hints, maybe we will feel more comfortable and we would be able to express ourselves better at that time.
The second common strategy identified was avoiding the use of negative comments or derogatory statements or a snubbing attitude in order to humiliate students. Most students agreed that this practice led to a decrease in their confidence and caused further uncertainty and confusion. An encouraging and welcoming attitude and interactive teaching was appreciated by students.

Respondent $A Q$ (student): 'As for the faculty members, there shouldn't be any negative comments from them, this lowers your confidence level. Faculty members should have an encouraging body language, they should be welcoming and they should try to extract answers from students. If the student is blank or nervous due to their attitude, then his confidence level will be shattered and his learning will also be hampered.'

Respondent $F$ (student): teachers do give off their own implicit reactions which are connotations of bad or negative reactions in that moment like smirking or frowning or making faces when we do give answers and that does put the students' confidence down a little bit.

Respondent AM (student): but they should not shatter the student in front of the patient as they also have their own self esteem. They should also not insult the students on the spot.

Lastly, there was discussion on constructive feedback, positive reinforcement, encouraging reflection, mentorship and counselling. All these were considered to be important for the mental well-being of students which would further help them learn by increasing their motivation.

Respondent A (student): 'As far as teachers go, if they give destructive criticism, it will only shatter the confidence of students, right. Their aim has to be to give constructive criticism, which, as already pointed out by QS, majority of teachers do go for constructive criticism.'

Respondent AH (student): 'They should also give positive feedback highlighting on the positives 
and help students improve their corrections.'

Respondent T (faculty): 'I give positive reinforcement by using words such as 'very good', 'this is correct' or 'go ahead'; if they are wrong you rephrase the question in such a way that they reach the correct answer. As faculty you should be very patient and calm and avoid giving gestures that would create more uncertainty, create a positive learning atmosphere where students are not afraid to speak up and discuss and they are not afraid of being wrong. In my opinion, mentorship also has a strong role here; they should be taught how to reflect so they can reflect on the situation and identify their needs.

Students also appreciated the ability of faculty members to identify their weak areas and work on them. Moreover, in instances where faculty members offered extra help or extra time after classes to students who had any queries or confusions greatly encouraged students and gave them a sense of comfort.

Respondent TZ (student): some of them try to stay after the ward timing so they can give students tips on how to do stuff better, which actually helps if you stay after the ward for 5-10 minutes after the ward timings. That helps a lot, which can be pretty encouraging for a student who actually wants to learn.

Majority of the students said they felt low on confidence, disappointed and depressed when they were not certain in their decision making. It was demeaning for them when their confidence shattered and they were treated like they were not doctors at all as it undermined their clinical acumen. Moreover, they felt inadequate and incompetent as they were unable to answer in front of the consultant, which led to them having doubts about themselves and many a times embarrassed to face the teacher again. This feeling was also influenced by the attitude of the teachers; some students also described a positive outcome as their teachers provided valuable feedback, which helped them learn better. One interesting aspect was put forward by a faculty member who was of the view that uncertainty is a drive to improve oneself and that it was crucial to ignite the thinking process in students. This could help them become safe practitioners as they would not be over confident.

Respondent FM (faculty): So, I think igniting uncertainty into the minds of students always ignites the thinking process. It makes a physician more safe when he is a little uncertain because then he is not over confident.

Respondent $F$ (faculty): As far as students are concerned, they become even more under confident, panicky and shaky, they will exhibit gestures of under confidence and this will be carried in the future in the well, which will make them under confident doctors.

\section{DISCUSSION}

Clinical rotations during clerkship years allow students hands on experience with real life situations to enhance their knowledge and facilitate competence in designated skills and management of problems. The present study revealed that the most common factor leading to uncertainty is that there is a lack of adequate clinical exposure, practice and rehearsal which leads to a lack of opportunity to acquire the necessary skills to be a competent doctor, resulting in a limited educational experience. This underexposure is supported by evidence in literature, where a significant correlation is seen between the frequency of a procedure performed and confidence levels of a student. ${ }^{15}$ The more the students are given exposure and practice, the more confident and certain they will be. It is seen that hands on experience in clinical wards is a significant predictor for building students' confidence. ${ }^{16}$ Other reasons influencing uncertainty stated in the current research were: non-serious attitude of students, lack of interest, stakes of exam, fear or being wrong or embarrassed in front of colleagues, attitude of teacher, previous experience and the practice of studying towards the end for an exam. In comparison, factors mentioned in literature encompass those experienced by physicians mainly such as variation in signs and symptoms of patients, physician's knowledge and skills, 
demands related to the disease, communication skills, and lastly, haste in medical consultations or errors in interpretation. ${ }^{7}$

A detailed analysis of the results of the study, unveiled the strategies to decrease uncertainty in decision making in medical students and to ensure a smooth learning process. It is essential to acknowledge and address this as expressing uncertainty is often suppressed by students due to a competitive environment where students have to constantly prove themselves or due to a fear of embarrassment or to deflect criticism. Masking this uncertainty can have harmful consequences in real patient encounters; handoff of patient information is an essential part of continuous patient care and it is crucial for the patient's safety that the next physician taking over correctly perceives the information in the handoff. In case of an inability to detect the implicit uncertainty, it can result in faulty clinical reasoning, thus causing medical errors. ${ }^{17}$

There is a lack of a structured format for oral case presentations, where feedback is usually unstructured, depending on the topic, personality of the teacher, time constraints and errors made by students. ${ }^{18}$ It is suggested that explicit instructions be given to students for the desired format and expectations, combined with structured feedback; this will help students refine their knowledge and clinical reasoning on a daily basis. Faculty members must also be able to interpret information presented, indulge in their own clinical reasoning and then form a diagnosis and management plan. A comparison of the students' interpretations will allow faculty members to identify their learning needs and tailor instruction. Repeated practice of case presentations fosters optimized learning, increases experience, improves clinical reasoning and facilitates assessment decisions. ${ }^{19}$

Supervisors should guide students on how to present long cases effectively; they should be weaved into a coherent narrative that encapsulates the main events leading to the patient presentation, a convincing argument, proper organization of facts and fluency in speaking are expected in a good presentation. ${ }^{18}$ Direct observation of students provides a valuable template to provide meaningful feedback which helps in reinforcing clinical skills and corrects deficiencies; students report their confidence levels are significantly influenced by the number of direct observations, more the observations, higher the confidence. ${ }^{20}$ Feedback should be given enthusiastically, with a positive and corrective attitude, while also working to meet student's individual needs. ${ }^{21}$ It should also be constructive, involving pointing out of diagnostically meaningful information, identification of irrelevant findings, highlighting the differentiating features and the importance for drawing conclusions for the correct diagnosis. ${ }^{22}$

Medical students often find uncertainty during case presentations a major source of stress. Students who are unable to tolerate medical uncertainty are seen to exhibit negative attitudes as they feel uncertain during clinical reasoning. ${ }^{23}$ It is suggested that a longitudinal clinical reasoning course is implemented in the curriculum, which should be done after training faculty members. ${ }^{24}$ Clinical teachers must guide students so as to facilitate clinical skills and reasoning of students; this guidance will promote their progress towards independence in wards. They can use various strategies to enhance the development of strong clinical reasoning skills; in case of a disorganized case presentation, they can give hints or prompts to the student to formulate a short summary of the case. They can also help students and make it a more interactive experience by reasoning aloud and highlighting the main features of the case; they should encourage students to compare and contrast different scenarios by creating linkages between their findings, thus bolstering their clinical acumen. Additionally, they should also encourage reading, to stimulate conceptualization as opposed to memorization, thereby reinforcing the significance of self-directed learning. ${ }^{22}$ Faculty members should not use negative comments or humiliate students as students feel embarrassed when their lack of knowledge is made known to all or when they are asked questions with the intent of causing distress to the student. ${ }^{25}$ Student support and counselling services are deemed effective in reducing student's anxiety. ${ }^{26}$ 
In order to reduce uncertainty in decision making, most participants suggested increased practice and exposure to patient interactions as students had earlier admitted to not being regular in their clinical ward rotations. This increased practice will help students become less didactic and more experiential; learning is contextualized and integrated with more hands-on experience; it allows students to make decisions, manage risks and cope with uncertainty. Moreover, it helps them understand their professional roles better, enhance their skills, improve their communication with patients, acquire research and presentation skills, critically evaluate data and apply evidencebased medicine principles in their case presentations. As a result, students not only work diligently, but also develop a sense of themselves by learning in different contexts of care while at the same time acquiring experience that makes them proficient enough in the tasks of qualified junior doctors. ${ }^{21}$ The most important impact of increased clinical exposure and practice is an increase in confidence levels of students. ${ }^{27}$

There needs to be an emphasis on improving communication skills and interpersonal skills as these are considered as a core competency for medical students; this helps in improved understanding, adherence to the advised treatment plans, resolution of symptoms, better decision making, and decreased incidence of malpractice claims. ${ }^{28}$ Meticulous history taking, building partnerships with patients, critical appraisal of current literature and reassessment of care also lead to a reduction in uncertainty. ${ }^{7}$

A positive learning environment with a culture of respect in the institute, and the construction of effective mentorship are seen to improve personal well-being of students, where faculty members are always ready to answer students' queries. ${ }^{23}$ It also nurtures deep self-directed learning and consequently good medical practice and is associated with increased student satisfaction, achievements and success. ${ }^{29}$ As mentioned in the results, reflective writing is supported by evidence in helping students tolerate uncertainty, thus strengthening their learning process. ${ }^{7}$
Students in this study mentioned how they take help from fellow peers to make their concepts clear and to improve their clinical acumen, which is also an effective strategy as mentioned in literature. ${ }^{30}$ Peer assisted learning involves students from the same program to help each other by taking joint responsibility for identifying and managing their learning needs; this method embraces a wide variety of activities that are undertaken by students, such as collaborative work projects, or discussion seminars. Learning experiences in PAL are reciprocal, benefitting all participants as it promotes critical thinking and active engagement of learners, making it an effective tool for learning in medical education. ${ }^{31}$

\section{CONCLUSION}

Uncertainty is an intrinsic aspect of medical education that can be faced by students at any stage of patient interaction during their oral case presentations. This can have implications on both students and patients, therefore, it is essential to identify the factors that contribute to this uncertainty in decision making and the strategies that can be used to reduce uncertainty to allow students to become competent and confident physicians.

\section{Copyright (C 12 June, 2021.}

\section{REFERENCES}

1. Han PKJ, Klein WMP, Arora NK. Varieties of Uncertainty in Health Care: A Conceptual Taxonomy. Med Decis Mak. 2011; 31(6):828-38.

2. McGovern R, Harmon D. Patient response to physician expressions of uncertainty: A systematic review. Ir J Med Sci. 2017; 186(4):1061-5.

3. Cousin G, Schmid Mast M, Jaunin-Stalder N. When physician-expressed uncertainty leads to patient dissatisfaction: A gender study. Med Educ. 2013; 47(9):923-31.

4. CookeS, Lemay JF. Transforming medical assessment: Integrating uncertainty into the evaluation of clinical reasoning in medical education. Acad Med. 2017; 92(6):746-51.

5. Lingard L, Garwood K, Schryer CF, Spafford MM. A certain art of uncertainty: Case presentation and the development of professional identity. Soc Sci Med. 2003; 56(3):603-16. 
6. Prince KJAH, Boshuizen HPA, Van Der Vleuten CPM, Scherpbier AJJA. Students' opinions about their preparation for clinical practice. Med Educ. 2005; 39(7):704-12.

7. Nevalainen MK, Mantyranta T, Pitkala KH. Facing uncertainty as a medical student-A qualitative study of their reflective learning diaries and writings on specific themes during the first clinical year. Patient Educ Couns. 2010; 78(2):218-23.

8. Blanch DC, Hall JA, Roter DL, Frankel RM. Is it good to express uncertainty to a patient? Correlates and consequences for medical students in a standardized patient visit. Patient Educ Couns. 2009; 76(3):300-6.

9. Luther VP, Crandall SJ. Commentary: Ambiguity and uncertainty: Neglected elements of medical education curricula? Acad Med. 2011; 86(7):799-800.

10. Duru E, Balkis M. The roles of academic procrastination tendency on the relationships among self doubt, self esteem and academic achievement. Egit ve Bilim. 2014; 39(173):274-87.

11. Holmes SM, Ponte M. En-case-ing the Patient: Disciplining Uncertainty in Medical Student Patient Presentations. Cult Med Psychiatry. 2011; 35(2):16382.

12. Politi MC, Clark MA, Ombao H, Dizon D, Elwyn G. Communicating uncertainty can lead to less decision satisfaction: A necessary cost of involving patients in shared decision making? Heal Expect. 2011; 14(1):84-91.

13. Lambert V a., Lambert CE. Qualitative Descriptive Research: An Acceptable Design. Pacific Rim Int J Nurs Res [Internet]. 2013; 16(4):255-256. Available from: http://antispam.kmutt.ac.th/index.php/PRIJNR/ article/download/5805/5064.

14. Kiger ME, Varpio $L$. Thematic analysis of qualitative data: AMEE Guide No. 131. Med Teach [Internet]. 2020; 0(0):1-9. Available from: https://doi.org/10.1080 /0142159X.2020.1755030.

15. Barr J, Graffeo CS. Procedural Experience and Confidence among Graduating Medical Students. J Surg Educ [Internet]. 2016; 73(3):466-73. Available from: http://dx.doi.org/10.1016/j.jsurg.2015.11.014.

16. Morgan PJ, Cleave-Hogg D. Comparison between medical students' experience, confidence and competence. Med Educ. 2002; 36(6):534-9.

17. Gärtner J, Berberat PO, Kadmon M, Harendza S. Implicit expression of uncertainty - Suggestion of an empirically derived framework. BMC Med Educ. 2020; 20(1):1-8.
18. Chan MY. The oral case presentation: Toward a performance-based rhetorical model for teaching and learning. Med Educ Online. 2015; 20(1).

19. Melvin L, Cavalcanti RB. The oral case presentation a key tool for assessment and teaching in competencybased medical education. JAMA - J Am Med Assoc. 2016; 316(21):2187-8.

20. Chen W, Liao S, Tsai C, Huang C, Lin C, Tsai C. Clinical skills in final-year medical students: the relationship between self-reported confidence and direct observation by faculty or residents. Ann Acad Med Singapore. 2008;37.

21. Dornan T, Tan N, Boshuizen H, Gick R, Isba R, Mann $\mathrm{K}$, et al. How and what do medical students learn in clerkships? Experience based learning (ExBL). Adv Heal Sci Educ. 2014; 19(5):721-49.

22. Bowen JL. Educational strategies to promote clinical diagnostic reasoning. N Engl J Med. 2006; 355(21):2217-25.

23. Kim $\mathrm{K}$, Lee $\mathrm{Y}-\mathrm{M}$. Understanding uncertainty in medicine: Concepts and implications in medical education. Korean J Med Educ. 2018; 30(3):181-8.

24. Harendza S, Krenz I, Klinge A, Wendt U, Janneck M. Implementation of a clinical reasoning course in the internal medicine trimester of the final year of undergraduate medical training and its effect on students' case presentation and differential diagnostic skills. GMS J Med Educ. 2017; 34(5):1-13.

25. Markman JD, Soeprono TM, Combs HL, Cosgrove EM. Medical student mistreatment: Understanding 'public humiliation.' Med Educ Online [Internet]. 2019; 24(1). Available from: https://doi.org/10.1080/10872981 .2019 .1615367 .

26. Khoshhal KI, Khairy GA, Guraya SY, Guraya SS. Exam anxiety in the undergraduate medical students of Taibah University. Med Teach [Internet]. 2017; 39(0):S22-6. Available from: http://dx.doi.org/10.1080/ $0142159 X .2016 .1254749$.

27. Chen W, Liao SC, Tsai $\mathrm{CH}$, Huang CC, Lin $\mathrm{CC}$, Tsai $\mathrm{CH}$. Clinical skills in final-year medical students: The relationship between self-reported confidence and direct observation by faculty or residents. Ann Acad Med Singapore. 2008; 37(1):3-8.

28. Zick A, Granieri M, Makoul G. First-year medical students' assessment of their own communication skills: A video-based, open-ended approach. Patient Educ Couns. 2007; 68(2):161-6. 
29. Veerapen K, McAleer S. Students' perception of the learning environment in a distributed medical programme. Med Educ Online. 2010; 15(9):1-11.

30. Gheihman G, Johnson M, Simpkin AL. Twelve tips for thriving in the face of clinical uncertainty. Med Teach [Internet]. 2020; 42(5):493-9. Available from: https://doi. org/10.1080/0142159X.2019.1579308.
31. Guraya SY, Abdalla ME. Determining the effectiveness of peer-assisted learning in medical education: A systematic review and meta-analysis. J Taibah Univ Med Sci [Internet]. 2020; 15(3):177-84. Available from: https://doi.org/10.1016/j.jtumed.2020.05.002.

\section{AUTHORSHIP AND CONTRIBUTION DECLARATION}

\begin{tabular}{|c|l|l|l|} 
No. & \multicolumn{1}{|c|}{ Author(s) Full Name } & \multicolumn{1}{|c|}{ Contribution to the paper } & Author(s) Signature \\
\hline 1 & Shahmain Shahzad & $\begin{array}{l}\text { Main author (Corresponding } \\
\text { author) } \\
\text { Co-Author }\end{array}$ & \\
\hline 2 & Fahad Ismail & & \\
\hline
\end{tabular}

